\title{
Recurrent Chylous Pericardial Effusion and Left Neck Mass
}

\author{
Denis Gilmore $^{1}$ Yolonda L. Colson ${ }^{1}$ \\ ${ }^{1}$ Department of Thoracic Surgery, Brigham and Women's Hospital, \\ Boston, Massachusetts, United States \\ Thorac Cardiovasc Surg 2012;60(S2):e25-e27.
}

\author{
Address for correspondence Denis Gilmore, M.D., Department of \\ Thoracic Surgery, Brigham and Women's Hospital, \\ 75 Francis Street, Boston, MA 02115, United States \\ (e-mail: dgilmor1@bidmc.harvard.edu).
}

\begin{abstract}
Keywords

- generalized lymphatic anomaly

- chylous effusion

We present a case of a woman initially presenting with a neck mass and subsequently found to have generalized lymphatic anomaly with diffuse lymphatic dilation and proliferation involving the mediastinum and pericardium. Intrathoracic involvement of generalized lymphatic anomalies can lead to recurrent pleural effusions, pericardial effusions, and severe respiratory infections. These anomalies cannot be cured and significantly impact quality of life. Multidisciplinary involvement is crucial to maximize symptom relief as surgery to remove lymphatics can be morbid and the disease is usually recurrent.
\end{abstract}

\section{Introduction}

Generalized lymphatic anomaly (GLA) is an uncommon disease of lymphatic malformation leading to dilatation and proliferation of lymphatic channels. This disease normally occurs in childhood characterized by symptoms of lymphatic congestion in areas of involvement. ${ }^{1,2}$ Severity of the clinical course is variable and difficult to treat. Intervention is aimed at symptom management rather than cure. Treatment options depend on sites involved but include combinations of surgery, interventional radiology, and/or chemotherapeutics. Treatment becomes difficult due to the progressive nature of the disease and adaptability of the lymphatic system; since once an area is treated, secondary lymphatic channels often develop, leading to new collections and symptoms.

\section{Case Presentation}

A 48-year-old woman presented with nonspecific precordial pain. She was briefly treated for bronchial asthma without relief. Initially, echocardiogram, pulmonary function tests, and computed tomography (CT) chest were normal. Over the following year, she noticed a neck mass which fluctuated in size. The mass was often stable for 2 to 3 weeks before increasing in size over days and then spontaneously decom- pressing over 2 to 5 days. Over this same time period, she began experiencing fatigue, dyspnea on exertion, and precordial chest pain. A neck magnetic resonance imaging (MRI) was performed demonstrating a $4.0 \times 4.0 \times 1.8 \mathrm{~cm}$ cystic left neck mass, thought to represent a second brachial cleft cyst. Her past medical history was significant for pericardiocentesis 1 year prior for $750 \mathrm{~mL}$ of cloudy serosanguineous fluid with subsequent analyses unrevealing. The pericardial effusion recurred within 3 weeks and she underwent subxiphoid pericardial window with $1200 \mathrm{~mL}$ of fluid evacuated. No further interventions were performed.

Following consultations with her otolaryngologist and cardiologist, she was scheduled for excision of this second brachial cleft cyst. Before surgery, a transthoracic echocardiogram was performed for symptoms of dyspnea on exertion which noted recurrence of pericardial effusion. She underwent pericardiocentesis with drain placement yielding $380 \mathrm{~mL}$ of chyle. To further evaluate this effusion and possible connection with the left neck mass, MRI and subsequent lymphangiogram were obtained. An extensive mediastinal lymphatic malformation, which extended superiorly to the left neck mass, was identified. The radiographic appearance of multiple mediastinal lymphatic channels communicating with the large lymphocele in the left neck and multiple lymphatic "pools" within the spleen and vertebra are all received

May 6, 2012

accepted after revision

July 8,2012

published online

November 19, 2012 (c) 2012 Georg Thieme Verlag KG Stuttgart · New York
DOI http://dx.doi.org/ $10.1055 / \mathrm{s}-0032-1326777$. ISSN 2194-7635. 

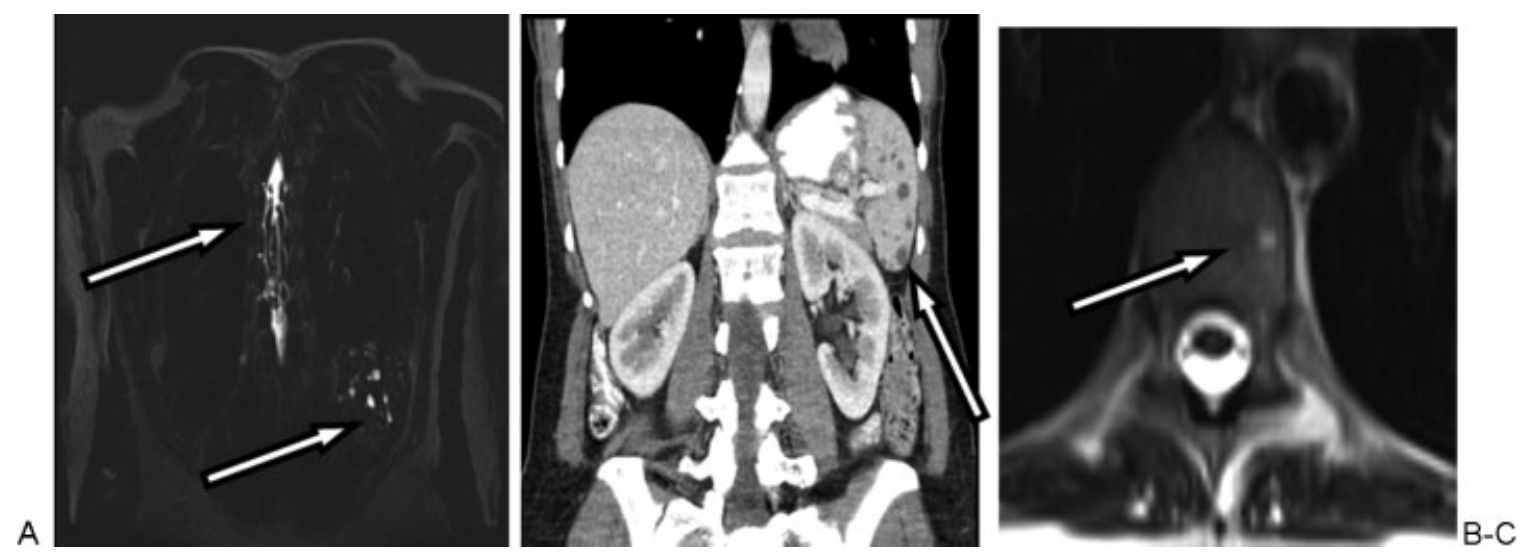

Figure 1 (A) Coronal MRI chest with 3D echo gradient sequences highlighting multichannel lymphatic anomalies within mediastinum and spleen. (B) Coronal CT abdomen showing lymphatic collections throughout the spleen. (C) Axial T2-weighted MRI chest demonstrating lymphatic dilatation in the vertebral body. CT, computed tomography; MRI, magnetic resonance imaging; 3D, three-dimensional.

consistent with diagnosis of GLA (-Fig. 1). Given prior pericardial window and extensive nature of lymphatic channels arborizing throughout the mediastinum, sclerotherapy and coil embolization, rather than extensive surgical resection with high risk of subsequent chronic chylothorax, were performed. The patient underwent intralymphatic injection of 15 units of bleomycin and placement of 14 coils with an excellent lymphangiographic result (-Fig. 2). Following this, drainage of pericardial fluid decreased significantly and the drain was removed. A loculated pericardial effusion has remained stable, without progression or intervention, over the current 14 months of follow-up.

\section{Discussion}

GLA is a rare congenital disease resulting in malformation of lymphatic vessels involving multiple sites of soft tissues, lungs, abdominal organs, or bones. Multiple terms are associated with GLA including: lymphangiomatosis, generalized lymphangioma, systemic cystic angiomatosis, multiple lym-

A
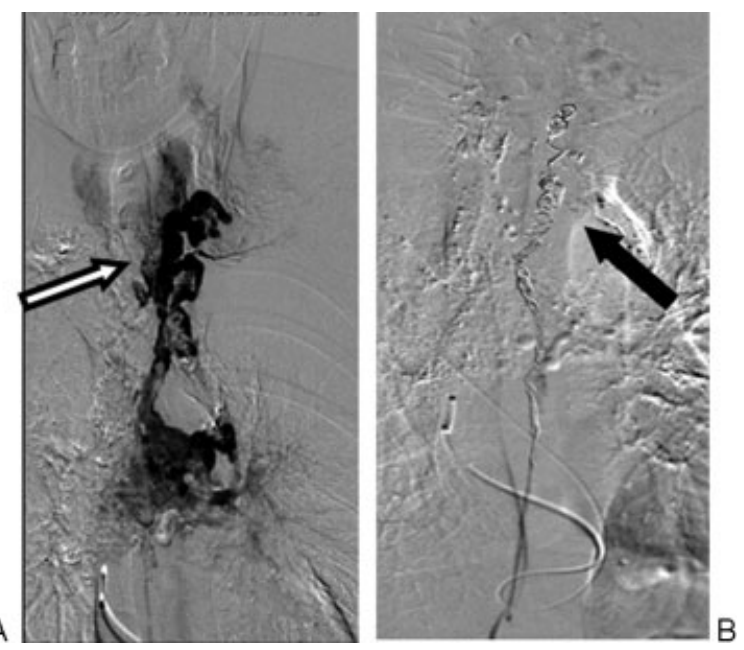

Figure 2 Lymphangiogram demonstrating connection of neck and mediastinal lymphatic anomalies (A) before coilization of lymphatic channels and obliteration of channels after (B). phangiectasias, and generalized lymphatic malformation. ${ }^{2}$ Lymphatic anomalies are thought to arise from disordered lymphatic development resulting in proliferation of lymphatic channels of varying size, complexity, and clinical course without definitive treatment options. ${ }^{2,3}$ The etiology is unknown but thought to occur during embryologic lymphatic development or with possible hormone connection. ${ }^{3}$ Greater than $60 \%$ of patients present in the first decade of life with symptoms and nearly all patients are identified by 20 years of age. ${ }^{4}$ Late presentation, as in this case, is rare and generally carries a better prognosis. The rate of disease progression differs in adult and pediatric populations, with adults exhibiting slower progression. Diagnosis is often made via clinical presentation alone. Histopathology, when available, demonstrates anastomosing endothelial lined spaces along lymphatic routes, accompanied by asymmetrically spaced bundles of spindle cells. Factor VIII-related antigen and cluster of differentiation 31 are endothelial markers that are useful in immunohistochemical staining of these channels. ${ }^{5}$ Multiple treatment options for generalized lymphatic anomalies are available but with varying success. As a result, standard treatment algorithms have not yet been determined. Surgical therapy is reserved for disease confined to a single, limited area or palliative in the cases of progressive symptoms; since once an area is treated, secondary lymphatic channels develop, leading to new symptomatic collections due to the wide abnormal network of lymphatic channels. Surgical options may involve radical resection of the affected area with potential for high morbidity and mortality. Conservative approaches remain first line treatment for wide spread disease with multiorgan involvement. These options include lymphatic embolization with sclerotherapy, drainage, radiotherapy, thoracic duct ligation, organ transplantation, systemic chemotherapy, and interferon therapy, although all have demonstrated suboptimal results. Standard follow-up of these patients has not been established. Often, clinical examination and symptoms guide the need for further imaging. $^{2-4,6,7}$ The prognosis, particularly in children, is usually poor as there is no cure for this generally progressive disease and treatment is focused on palliative symptom relief. GLA 
requires multidisciplinary approach secondary to its progressive nature. Treatment options are numerous but with only varying success. Symptomatic relief should be the immediate goal of care but decisions need to consider long-term morbidity and future treatment options.

\section{References}

1 Margaf LR. Thoracic lymphangiomatosis. Pediatr Pathol 1996;16: 155-160

2 Faul JL, Berry GJ, Colby TV, et al. Thoracic lymphangiomas, lymphangiectasis, lymphangiomatosis, and lymphatic dysplasia syndrome. Am J Respir Crit Care Med 2000;161(3 Pt 1):1037-1046
3 Rostom AY. Treatment of thoracic lymphangiomatosis. Arch Dis Child 2000;83(2):138-139

4 Ozeki M, Funato M, Kanda K, et al. Clinical improvement of diffuse lymphangiomatosis with pegylated interferon alfa-2b therapy: case report and review of the literature. Pediatr Hematol Oncol 2007;24(7):513-524

5 Rosai J. Lymph vessels. In: Rosai J, ed. Ackerman's Surgical Pathology. St. Louis: Mosby; 1995:2221-2226

6 Zisis C, Spiliotopoulos K, Patronis M, et al. Diffuse lymphangiomatosis: are there any clinical or therapeutic standards? J Thorac Cardiovasc Surg 2007;133(6):1664-1665

7 Raman SP, Pipavath SN, Raghu G, Schmidt RA, Godwin JD. Imaging of thoracic lymphatic diseases. AJR Am J Roentgenol 2009;193(6): 1504-1513 